East African Medical Journal Vol. 87 No. 1 January 2010

DENTAL CARIES, GINGIVITIS AND THE TREATMENT NEEDS AMONG 12-YEAR-OLDS

R. O. Owino, BDS, MDS, Tutorial Fellow, M.A. Masiga, BDS, MSc, Senior Lecturer, P.M. Ng'ang'a, BDS, MSD, PhD, Associate Professor, Department of Paediatric Dentistry and Orthodontics and F. G. Macigo BDS, MPH, PGD-STI, Senior Lecturer, Department of Periodontology and Community Dentistry, School of Dental Sciences, University of Nairobi, P.O. Box 19676 - 00202, Nairobi, Kenya

Request for reprints to: Dr. R. O. Owino, Department of Paediatric Dentistry and Orthodontics, School of Dental Sciences, University of Nairobi, P.O. Box 19676 - 00202, Nairobi, Kenya

\title{
DENTAL CARIES, GINGIVITIS AND THE TREATMENT NEEDS AMONG 12-YEAR-OLDS
}

\author{
R. O. OWINO, M.A. MASIGA, P.M. NG'ANG'A and F. G. MACIGO
}

\begin{abstract}
Background: One of the World Health Organisation (WHO) global health goals stated that the global average for dental caries should not have been more than 3 DMFT at 12 years of age by the year 2000. However, the prevalence of dental caries, gingivitis and dental treatment needs among 12-year-old children in many regions of the developing world had then hardly been investigated.

Objective: To determine the prevalence of dental caries and gingivitis and the dental treatment needs among 12-year-old children in Kitale Municipality in North-Western Kenya.

Design: Descriptive cross-sectional study.

Setting: Primary schools in Kitale Municipality.

Subjects: Two hundred and ninety two children aged 12 years were randomly selected from eight schools that were also randomly selected. Data were collected according to the WHO Oral Health Survey Methods. The children were examined for decayed (D), missing due to decay (M) and filled teeth and the caries experience using the DMFT index was computed. Gingivitis and treatment needs were assessed using the CPITN index.

Results: The overall prevalence of dental caries was $50.3 \%$ while its prevalence when only the permanent teeth were considered was $44.5 \%$ with a mean DMFT of $0.92+/-50$ 1.36. Girls had a significantly higher caries experience (DMFT) than boys $(p<0.05)$. The main treatment need indicated for decayed teeth was one surface restoration with $46.9 \%$ of all the children requiring this kind of treatment. Endodontics was indicated in $7.5 \%$ of the children while extractions were the least required treatment with $5.1 \%$ of the children examined requiring this treatment. The prevalence of gingivitis was $77.7 \%$. Out of these $38.7 \%$ of the children required professional dental care entailing scaling and oral prophylaxis.

Conclusion: The prevalence of dental caries and gingivitis was high hence there was greater need for one surface restoration and full mouth scaling for these children. School based oral health programme to improve oral hygiene of these children is recommended.
\end{abstract}

\section{INTRODUCTION}

Oral diseases qualify as a major public health concern owing to their high prevalence and incidence in all regions of the world (1). Dental caries and gingivitis are the two most common dental diseases affecting children worldwide. These two diseases are, to a large extent, the result of the presence of dental plaque. Dental plaque is defined as an adherent structured mass that forms on the tooth surfaces and is composed primarily of micro-organisms (2).
The period of adolescence begins between 10 and 12 years ending at 20 years of age. At this stage there are progressive anatomical, physiological and mental changes in the child that are associated with new freedoms, responsibilities and social demands (3). The progression of dental caries and gingivitis are seen to reach a peak during middle adolescence (3). Therefore, the dental practitioners' knowledge of the prevalence of dental caries and gingivitis among 12-year-old children and their treatment needs would enable the formulation of an appropriate oral health 
management strategy for these children. One of the World Health Organisation (WHO) global oral health goals stated that the global average for dental caries would be no more than 3 DMFT at 12 years of age by the year 2000. Yet baseline data desirable for appropriate interventions were scarcely forthcoming then and even now among the less developed world populations including those in Kenya. Due to lack of baseline data on dental caries among 12-yearold children, it becomes very difficult to formulate oral health policies and goals for this region as recommended by WHO oral health goals for the year 2020 (4). These WHO oral health goals for 2020 are not meant to be prescriptive but meant to be tailored to suite the particular region, and their purpose is to encourage policy makers to think globally and act locally.

Although dental caries is not life threatening, it can be painful, disfiguring and expensive to manage. Thousands of dollars, if not millions, are spent by countries each year in restoring decayed teeth in addition to the numerous school/work hours that are lost (1). Yet dental caries is preventable by oral hygiene techniques, diet counselling and fluoride therapy. Dental caries arises from the consumption of fermentable carbohydrates in the diet. Sucrose has been described as the "arch criminal" in the aetiology of caries (5). Dental caries is the most prevalent oral disease in several Asian and Latin American countries but appears to be less common and less severe in African countries (1). Furthermore in the light of the changing living conditions, it is expected that the incidence of dental caries will increase in many African countries due to the growing consumption of sugars and inadequate exposure to fluorides (1).

Periodontal diseases are defined as those which attack the supporting structures of teeth (6). Periodontal diseases are a result of combined chemical, bacterial and mechanical irritation from dental plaque and calculus (7). There are two main types of periodontal diseases namely gingivitis and periodontitis. The term gingivitis is used to designate inflammatory lesions that are confined to the marginal gingivae. Once the lesions extend to include destruction of the connective tissue attachment of the tooth the disease is designated periodontitis. Epidemiological studies have shown the prevalence of gingivitis among children to be high (8-10). Caranza's report from the US national sample surveys 1986-1987 among 12-17-year-olds reported a prevalence of $58.8 \%$ for gingivitis (5). Al Banyan et al. (9) in 2000 found the highest reported prevalence of gingivitis of $100 \%$ among 5-12-yearold children in Riyadh Saudi Arabia. A study by Jose et al. (11) in 2003, however, revealed a very low prevalence of gingivitis of $15 \%$ among 12-15-year-old children in Kerala, India. Studies conducted in Africa have also revealed a high prevalence of gingivitis as high as $80 \%$ among 12-year-old children in South Africa. Regionally, in Ethiopia, a lower prevalence of gingivitis of $53.4 \%$ among 12-year-old children has been documented (13). Very few studies have been done on the prevalence of gingivitis among the 11-14-year-olds in Kenya.

Oral health treatment needs are demands by the population for oral healthcare and in the context of this study include the demand for treatment of dental caries and inflammatory periodontal disease (14). Nalweyiso et al. (15) in 2004 in Uganda found that $52.5 \%$ of the children needed a filling while $33 \%$ needed one or more teeth extracted. Al Banyan et al. (9) in 2000 found that $41 \%$ of children aged 512 years old in Saudi Arabia required treatment for dental caries in the permanent dentition. Data on dental treatment needs forms a basis for planning oral health programmes including estimating personnel requirements and the costs of an oral health programme under prevailing local conditions.

Although epidemiological studies on dental caries and gingivitis have been conducted in Kenya, they may not reflect the current situation. Dietary patterns have been changing with urbanisation, modernisation and industrialisation. There has been an increased marketing and consumption of Western type sweet snacks in the Kenyan market which could contribute considerably to changes in the prevalence of dental caries and periodontal disease (16). In all the epidemiological studies on dental caries and gingivitis carried out in Kenya among the 12-year olds, the main objective was to find out the prevalence of the disease. There are few studies on dental caries and gingivitis reported in Kenya (17) and none of these studies had investigated treatment needs for dental caries and gingivitis. The aim of this study was therefore, to investigate the prevalence of dental caries and gingivitis and the overall treatment needs among 12-year-old children in peri-urban Kitale, North Western Kenya.

\section{MATERIALS AND METHODS}

This was a descriptive cross-sectional study using primary school based study groups drawn from Kitale municipality.

Study population: The study population was defined as 12-year-old children attending primary schools in Kitale municipality.

Sampling methods: Kitale municipality has 26 public primary schools distributed in four educational zones namely; Bidii, Milimani, Grassland and Bondeni. Two schools were randomly selected from each of the four educational zones by simple random sampling, thus a total of eight schools were selected for this study. The number of children selected from each zone was 
based on the number of 12-yearold children in that zone as a proportion of the total number of 12 yearold-children enrolled in public primary schools (1668) multiplied by the calculated sample size of 288 . This followed that the different zones were represented as follows:

- Bidii zone $=402 / 1668 \times 288=69$ pupils.

- Milimani zone $=272 / 1668 \times 288=47$ pupils .

- Grassland zone $=402 / 1668 \times 288=69$ pupils .

- Bondeni zone $=592 / 1668 \times 288=102$ pupils .

Since two primary schools were selected from each zone, each school was represented by the calculated number of children for that zone divided by two. Therefore, 35 children per school were selected from Bidii and Grassland zone, 24 from Milimani zone and 51 from Bondeni zone. For each school a list of all 12 year old children with consent from parents was made using class attendance registers of standard. Every second 12-year-old child was examined from this list.

Data collection instruments and techniques: Two clinical oral health officers were selected to be field assistants and were trained on how to record the results of oral examination and on disinfection and sterilisation of dental instruments used. An electric autoclave and glutaraldehyde (cidex) were used to sterilise instruments. Intra-oral examination was carried out by one of the authors (ROO) under natural light in a classroom of the respective school with the child seated on a classroom chair. Gingivitis and treatment need was assessed using CPITN index.

However, due to lack of the appropriate CPITN probe, the Michigan ' $\mathrm{O}$ ' probe was used to examine the gingival health of the children and thus the protocol used in this study was a modification of the WHO protocol for examination of gingivitis. Dental caries was assessed using the criteria and methods recommended by the WHO (14). A structured data collection form adopted from that recommended by WHO was used for recording the results of dental caries examination. Each tooth was recorded as either decayed (D), missing due to decay (M) or filled (F). A Shepherd's Crook (number 23) dental probe was used to check for caries after drying the tooth with cotton gauze. The extent of seven caries to dentine and pulp was also determined by clinical examination. During data collection, 29 children were re-examined to determine intraexaminer variability. The intra-examiner Kappa values obtained were 0.87 for dental caries and 0.81 for gingivitis which showed good consistency and minimal variability.
Data analysis: Data were analysed using the SPSS version 12 (SPSS Inc., Chicago, IL, USA) computer software. To asses gender differences in caries experience and treatment needs, Chi-square tests of significance were computed with statistical significance pessed below 5\%. Mann Whitney U-test was used to find out the relationship between dental caries and gingivitis.

\section{RESULTS}

Among the 292 children aged 12 years who were examined, 140(47.9\%) were boys and 152(52.1\%) were girls. The overall prevalence of dental caries that included both permanent and deciduous teeth was $50.3 \%$. However, the prevalence of dental caries in the permanent dentition was $44.5 \%$.

The overall mean DMFT was $0.92 \pm 1.36$ with females having a higher caries experience (DMFT of $1.07 \pm 1.45$ ) than males (DMFT of $0.67 \pm 1.23$ ); and the difference was statistically significant $(\mathrm{p}<0.05 \mathrm{p}=0.042)$. The decayed component accounted for $95.5 \%$ of the DMFT while teeth missing due to decay accounted for $4.5 \%$. Only 16 children (12.2\%) had a DMFT of greater than three while 162 children $(55.5 \%)$ who were free of dental caries (Table1). The mandibular first permanent molar was the most commonly decayed tooth.

Table 1

Percentage distribution of DMFT scores among the 12year-old children in Kitale municipality

\begin{tabular}{lcc} 
DMFT & Frequency & $(\%)$ \\
\hline 0 & 162 & 55.5 \\
1 & 56 & 19.2 \\
2 & 38 & 13.0 \\
3 & 20 & 6.8 \\
4 & 10 & 3.4 \\
5 & 2 & 0.7 \\
6 & 2 & 0.7 \\
7 & 1 & 0.3 \\
8 & 1 & 0.3 \\
\hline Total & 292 & 100 \\
\hline
\end{tabular}

A majority of the children (46.9\%) required at least one surface restoration in their permanent teeth (Table 2). Very few children $(5.1 \%)$ required pulp therapy and extraction of teeth $(7.5 \%)$. 
Table 2

Dental caries treatment need among the 12 year-old-children in Kitale municipality

\begin{tabular}{|c|c|c|c|c|c|c|c|}
\hline Treatment need & \multicolumn{2}{|c|}{ Male $(\%)$} & \multicolumn{2}{|c|}{ Female $(\%)$} & Total & $(\%)$ & Statistical test \\
\hline One surface filling & 61 & 43.6 & 76 & 50.0 & 137 & 46.9 & $\begin{array}{l}X^{2}=0.059 ; 1 \mathrm{df} \\
P>0.05(0.808)\end{array}$ \\
\hline Extraction & 10 & 7.1 & 12 & 7.9 & 22 & 7.5 & $\begin{array}{l}X^{2}=1.209 ; 1 \mathrm{df} \\
P>0.05(0.271)\end{array}$ \\
\hline Pulp therapy & 8 & 5.7 & 7 & 4.6 & 15 & 5.1 & $\begin{array}{l}X^{2}=0.184 ; 1 \mathrm{df} \\
P>0.05 \quad(0.668)\end{array}$ \\
\hline
\end{tabular}

The prevalence of gingivitis among the children examined was $77.7 \%$ (Table 3). Only $22.3 \%$ did not show any signs of gingivitis. A small proportion of the children $(10.6 \%)$ had all the six sextants with a CPI score of one. As shown in Table 3 more females than males were affected by gingivitis but the difference was not statistically significant $(\mathrm{P}>0.05)$. There were $38.7 \%$ of the children with a CPI score of two (Table 4). This represented those children who had calculus or other plaque retentive factors. Only $2.1 \%$ of the children had all the quadrants affected by plaque retentive factors (Table 4). A large proportion of the children $(77.7 \%)$ required oral hygiene instructions, only $38.7 \%$ needed professional dental care while $22.3 \%$ did not require treatment. There was no difference in the periodontal treatment needs between boys and girls.

Table 3

Distribution of the 12-year-old children according to sextants with CPI score of one

\begin{tabular}{|c|c|c|c|}
\hline Sextant & Male $(\%)$ & Female $(\%)$ & Total $(\%)$ \\
\hline 0 & $\begin{array}{ll}28 & 43.1\end{array}$ & $\begin{array}{ll}37 & 56.9\end{array}$ & $\begin{array}{ll}65 & 22.3 \\
\end{array}$ \\
\hline 1 & 66.7 & $5 \quad 33.3$ & 5.1 \\
\hline 2 & 48.2 & $29 \quad 63.0$ & 19.2 \\
\hline 3 & 37.0 & 63.0 & 15.8 \\
\hline 4 & 50.0 & 50.0 & 19.9 \\
\hline 5 & 61.9 & 38.1 & 7.2 \\
\hline 6 & 51.6 & $15 \quad 48.4$ & $31 \quad 10.6$ \\
\hline Total & 47.9 & $\begin{array}{ll}152 & 52.1\end{array}$ & 292100 \\
\hline
\end{tabular}

Table 4

Distribution of the 12-year-old children according to sextants with a CPI score of two

\begin{tabular}{lllllll}
\hline Sextant & Male & $(\%)$ & \multicolumn{2}{l}{ Female $(\%)$} & Total & $(\%)$ \\
\hline 0 & 86 & 48.0 & 93 & 52.0 & 179 & 61.3 \\
1 & 19 & 50.0 & 19 & 50.0 & 38 & 13.0 \\
2 & 23 & 45.1 & 28 & 54.9 & 51 & 17.5 \\
3 & 5 & 33.3 & 10 & 66.7 & 15 & 5.1 \\
4 & 2 & 100.0 & 0 & 0.0 & 2 & 0.7 \\
5 & 1 & 100.0 & 0 & 0.0 & 1 & 0.3 \\
6 & 4 & 66.7 & 2 & 33.3 & 6 & 2.1 \\
\hline Total & 140 & 47.9 & 152 & 52.1 & 292 & 100 \\
\hline
\end{tabular}


Children who had gingivitis (CPITN $>0$ ) had a higher caries experience (DMFT $0.93 \pm 1.4)$ than children with no gingivitis $(\mathrm{CPITN}=0)$ who had a mean DMFT of $0.89 \pm 1.2$. However, this difference was not statistically significant ( $p>0.05 ; p=0.082$ ).

\section{DISCUSSION}

The overall prevalence of dental caries including both deciduous and permanent dentition was $50.3 \%$. The prevalence of dental caries in the permanent dentition was found to be high $44.5 \%$. This prevalence was higher than has been reported in previous studies amongst children in Nairobi $(18,19)$. However, the prevalence of caries in this study was not different from that reported by $\mathrm{Ng}^{\prime}$ ang'a et al. (20) in 1992 of $50 \%$ among 13-15-year-olds. These findings may be interpreted in two ways: either, the prevalence of dental caries in children in Kenya may be on the increase or there could be geographical/regional variation in the prevalence of dental caries as implied by Pitts et al. (21, 22). However, the sample size and diagnostic criteria in this study may have been different from the previous studies and, therefore, conclusions and comparisons may not be made objectively.

TheDMFT in thisstudy was well within the WHO recommended global average for dental caries for the year 2000 . However, with this data policy makers can formulate oral health goals for 2020 by determining the percentage reduction of the DMFT they wish for this region. This reduction must be realistic and should be based on the availability of financial support from the government, human resources, dental materials and equipment (4). The DMFT of these children was low considering that Kitale Municipality is classified as an urban centre. It is therefore important to note that although these children attended urban schools, $80 \%$ of them lived in their rural small scale farms outside the Municipality. Therefore, an explanation for the low caries experience may possibly be attributed to the low socio-economic status of the peasant farmers and the relative inability of their children to access and routinely indulge in cariogenic snacking. Socio-economic status has been shown to have an influence on the pattern of cariogenic snacking (23, 24). The decayed teeth had the greatest contribution to the caries experience accounting for $95.5 \%$ of the DMFT score. The level of untreated tooth decay was, therefore, high.

Untreated dental caries in adolescents has been shown to reflect low utilisation of dental services and may be a result of limited access to dental care or dental avoidance by parents and their children (25). Other reasons for untreated dental caries include lack of awareness by the parents and low socio-economic status. More recently the National Oral Health Policy document has indicated that dental clinics in most government hospitals are poorly equipped and understaffed (26). Dental restorative services were lacking and the only curative service available was tooth extraction. Dental restorative work in Kitale was only available at a private practitioner's clinic at a fee that may not be affordable. There was also lack of adequate medical personnel to carry out oral health promotion and preventive services.

Dental caries experience was higher in females than males $(p<0.05)$, an observation that has also been reported in other studies children in Kenya $(18,20,27)$. Although strong conclusions cannot be made on the reasons for this, it has been suggested that females may probably be involved in more cariogenic snacking than males (20). The eruption time of teeth in females occurs earlier than males by up to ten months as described by Manji et al. in 1985 (28) and Hassanali et al. in 1981 (29) and this could be a contributory factor as teeth in girls are exposed to the risk factors of caries much earlier. The first permanent molars had the highest caries incidence, and this was more in the mandibular molars than maxillary molars. The clinical crown morphology of these teeth including the occlusal grooves, pits and fissures are thought to make these teeth more susceptible to decay. Another factor that may make these teeth more prone to decay is the fact that they are the first permanent teeth to erupt and are exposed to the oral cavity at an earlier time compared to the other teeth. Several other studies have demonstrated that the occlusal surface of the first permanent molar is the site in the dentition which is most frequently attacked by dental caries $(30,31)$. This finding supports fissure sealing of newly erupted first molars as a preventive measure.

Majority of the children $(46.9 \%)$ required one surface restoration, $5.1 \%$ of the children required pulp treatment while $7.5 \%$ required extractions. These treatment needs were higher than what was reported in Uganda where $30.2 \%$ of the children needed a filling while $6.4 \%$ required an extraction (15). This information suggests a high demand for restorative services. Since majority of the children required one surface restoration, atraumatic restorative treatment (ART) may be a viable option of treatment for these children. The poorly equipped dental facility in Kitale Municipality and the scarcity of dentists justify the introduction of this ART which has been shown to have an improved three year survival rate of between $79 \%$ and $88 \%$ (32- 34).

The prevalence of gingivitis was $77.7 \%$ considering those children who had a CPI score of at least one. This is a high prevalence indicating that periodontal disease in children may be a major public health concern in Kitale Municipality. The prevalence of gingivitis in this study though high, was, however, lower than that previously reported in other local studies $(8,35)$. This finding of a high prevalence of 
gingivitis is comparable to other studies conducted among children in Africa and other parts of the world $(9,10,12)$. However, direct comparison of the results should be done with caution because different indices, diagnostic criteria and methods of examination may have been used in the different studies.

The impact of a high prevalence of gingivitis is mainly two-fold. First, studies have shown that there is a relationship between oral hygiene/gingivitis and caries experience. This study has shown that the dental caries experience was higher in children with gingivitis than those without gingivitis. Tucker et al. (36) in 1976 demonstrated that dental caries experience increments were lower in children with good oral hygiene when followed-up for three years as opposed to those with poor oral hygiene and severe gingivitis. Therefore, with this high prevalence of gingivitis it may be expected that the caries experience could increase if preventive measures are not put in place. Secondly gingivitis could lead to the destruction of the tooth supporting structures followed by tooth loss (6). In this study $77.7 \%$ of the children required oral hygiene instructions only as a treatment need while $38.7 \%$ required full mouth scaling. Therefore, there is need for oral health preventive programmes in this population which may be achieved by integrating oral health into primary health care as is the case in other developing countries (37).

In conclusion, the present investigation indicates that prevalence of dental caries and gingivitis was high hence there was greater need for one surface restoration, oral hygiene instructions and motivation and full mouth scaling for these children. It is recommended that school based oral healtheducation programme be instituted to improve oral hygiene status of these children.

\section{ACKNOWLEDGEMENTS}

We thank the School of Dental Sciences and Colgate Palmolive for part sponsorship of this research. We would also like to thank Prof. M. L. Chindia and C. Kinuthia for the valuable contribution in preparation of this manuscript.

\section{REFERENCES}

1. Peterson, E. K. The World Oral Health Report 2003: Continous improvement of oral health in the 21st century the approach of the WHOGlobal Oral Health Programme. Comm. Dent. Oral. Epidemol. 2003; 31: 3-23.

2. Baer, P.N. and Benjamin, S.D. Periodontal diseases in children and adolescents. $1^{\text {st }}$ edition, J.B Lippincott: Philadelphia. 1974; pp: 287-290.

3. David, W. A. Jnr. The adolescent patient; 2nd edition CV Mosby: Saint Louis. 1970; pp: 61-69.
4. Hobdell, M., Petersen, P.E. and Johnson, N. Global goals for oral health 2020. Int. Dent. J. 2003; 53: 285288.

5. Newbrun, E. Sucrose, the arch criminal of dental caries. Odontol. Revy. 1967; 18: 373-386.

6. Irving Glickman: Clinical periodontology, 3rd edition. W. B. Saunders: Harcourt. 1966; pp: 11-19.

7. Ray, H.G. and Orban, B. Deep necrotic foci in the gingiva. J. Periodont. 1948; 19: 91-95.

8. Manji, F. Gingivitis, dental fluorosis and dental caries in primary school children of Nairobi, Kenya. East Afr. Med. J. 1984; 61: 524-532.

9. Al Banyan, A., Echeverri, E.A., Narendran, S. and Keene, H.J. Oral Health Survey of 5-12-year-old children of national guard employees in Riyadh, Saudi Arabia. Int. J Paed. Dent. 2000; 10: 39-46.

10. Mosha, H.J. Dental caries, gingivitis and oral hygiene among school children in Dar-es-Salaam - Tanzania. Odontostomatol. Trop. 1986; 91: 43-46.

11. Jose, A. and Joseph, M.R. Prevalence of dental health problems among school going children in rural Kerala. J. Indian Soc. Pedo. Prev. Dent. 2003; 21: 147-151.

12. Brindle, R., Wilkinson, D., Harrison, A. and Connolly, C. Oral health in Hlabisa, KwaZulu / Natal - a rural school and community based survey. Int. Dent. J. 2000; 50: 13-20.

13. Simon, C., Tesfaye, F. and Berhane, Y. Assessment of the oral health status of school children in Addis Ababa. Ethiopia. Med. J. 2003; 41: 245- 256.

14. World Health Organization; Oral Health Surveys, 4 th edition, WHO Publication: Geneva. 1997; pp: 21-46.

15. Nalweiso, N., Busingye, J., Whitworth,J. and Robinson, P.G. Dental treatment needs of children in a rural subcounty of Uganda. Int. J. Paed. Dent. 2004; 14: $27-33$

16. Ngatia, E. M. A study of dietary patterns, nutritional status and dental caries in pre-school children in Nairobi, Kenya. MSc. Thesis. 1998.

17. Ng'ang'a, P.M. An overview of epidemiologic and related studies undertaken on common dental diseases and conditions in Kenya between 1980- 2000. Afr. J. Oral. Hlth Sci. 2002; 1:103-110.

18. Manji, F., Mosha, H. and Frencken, J. The pattern of dental caries among 12 year old rural children in Kenya and Tanzania. East Afr. Med J. 1988; 65: 753- 758.

19. Dattani, S., Hawley, G.M. and Blinkhorn, A.S. Prevalence of dental caries in 12-13 year old Kenyan children in urban and rural areas. Int. Dent. J. 1997; 47: 355-357.

20. Ng'ang'a, P.M. and Valderhang, J. Dental caries in primary school children in Nairobi, Kenya. Acta. Odontol. Scand. 1992; 50: 269-272.

21. Pitts, N. B., Evans, D.J. and Nugent, Z.J. The dental caries experience of 12 year old children in the United Kingdom. Surveys Coordinated by the British Association for the study of community dentistry in 1996/97. Comm. Dental Hlth. 1988; 15: 49-54.

22. Pitts, N. B., Evans, D.J. and Nugent, Z.J. The dental caries experience of 12 year old children in the United Kingdom. Surveys Coordinated by the British Association for the study of community dentistry in 1998/99. Comm. Dental Hlth. 2000; 17: 48-53.

23. Manji, F. Dental caries and socio-economic factors amongst Nairobi School children. Odontostomatol. Trop. 1986; IX 3: 141-145. 
24. lrigoyen, M.E., Luengas, I.F., Yashine, A. and Mejia, A.M. Dental caries experience in Mexican school children from rural and urban communities. Int. Dent. J. 2000; 50:41-45.

25. Skaret, E., Weinstein, P., Milgrom, P., Kaakko, T. and Getz, T. Factors related to severe untreated tooth decay in rural adolescents: a case control study for public health planning. Int. J. Paed. Dent. 2004; 14:17-26.

26. Ministry of Health-Kenya. National Oral Health Policy and strategic plan: 2000-2012.

27. Sogi, G. and Bhasakar, D.J. Dental caries and oral health status of 13 to 14 year old school children of Davangere. J. Indian Soc. Pedo. Prev. Dent. 2001; 21:113-116.

28. Manji, F. and Mwaniki, D. Estimation of median age of eruption of permanent teeth in Kenyan African children. East. Afr. Med. J. 1985; 62: 252-260.

29. Hassanali, J. and Odhiambo, J.W. Ages of eruption of permanent teeth in Kenyan African and Asian children. Annals Human Biology. 1981; 8: 425- 431.

30. Silverstone, L.M., Johnson, N.W., Hardie, J.M and Williams, R.AD. Dental caries. Aetiology, pathology and prevention. The MacMillan Press Ltd; 1981; pp: 4-69.
31. Nikiforuk, G. Understanding Dental Caries, 1Etiology and Mechanisms; Karger: New York. 1985; pp:1-52.

32. Holmgren, C.J. and Frencken, J. E. Painting the future for ART. Comm. Dent. Oral. Epidemol. 1999; 27: 449453.

33. Wong, M.C.M., Schwarz, E., Lo, E.C.M. Patterns of dental caries severity in Chinese kindergarten children. Comm. Dent. Oral. Epidemol. 1997; 25: 343347.

34. Van'tHof, M.A, Frenken, J.,E.,Helderman, W.H.O and Holmgren, C.J. the atraumatic Restorative Treatment (ART) approach for managing dental caries: a metaanalysis. Int. Dent J. 2000; 56: 345-351.

35. Butt, M. H. Dental health of patients attending Nyeri Provincial dental clinic. Odontostomatol Trop. 1986; 2:107-113.

36. Tucker, G.J., Andlaw, R.J. and Burchell, C.K. Relationship between oral hygiene and dental caries incidence in 11-year-old children. A3 year study. Brit. Dent. J. 1976; 141: 75-79.

37. Helderman, W.H.P., Begum, A., Adyatmaka, A., et al. Integrating oral health into primary health careexperiences in Bangladesh, Indonesia, Nepal and Tanzania. Int. Dent. J. 1999; 49: 240-248. 